\title{
Chronic Hypertension, Perinatal Mortality, and the Impact of Preterm Delivery: A Population-Based Study in the United States, 2015-2018
}

\author{
Sonal Grover ${ }^{1}$, Justin Brandt ${ }^{1}$, Uma Reddy ${ }^{2}$, and Cande Ananth (STATS CONSULTS \\ ONLY) $)^{1}$ \\ ${ }^{1}$ Rutgers Robert Wood Johnson Medical School \\ ${ }^{2}$ Yale University School of Medicine
}

March 10, 2021

\begin{abstract}
Objective: To estimate the association between chronic hypertension and perinatal mortality and evaluate the extent to which this risk is impacted by preterm delivery. Design: Cross-sectional analysis. Setting: US, 2015-2018. Population: Singleton births from 20-44 weeks' gestation. Main outcomes and measures: We derived the risk of perinatal mortality in relation to chronic hypertension from fitting log-linear Poisson models with robust variance. Risk ratios (RR) and 95\% confidence intervals (CI) were estimated after adjusting for confounders. The impact of misclassifications and unmeasured confounding biases were assessed. Causal mediation analysis was performed to quantify the impact of preterm delivery on the association. Results: Of the 15,090,678 singleton births, perinatal mortality was 22.5 per 1000 births in chronic hypertensive pregnancies compared to 8.2 per 1000 births in normotensive pregnancies (adjusted RR 2.05, 95\% CI 2.00, 2.10). Corrections for exposure misclassification and unmeasured confounding biases substantially increased the risk estimate. Although, causal mediation analysis revealed that most of the effect of chronic hypertension on perinatal mortality was mediated through preterm delivery, the perinatal mortality rates were highest at early term, term, and late term gestations, suggesting that a planned early term delivery at 37-386/7 weeks may optimally balance risk in these pregnancies. Additionally, 87\% (95\% CI 84, 90) of perinatal deaths could be eliminated if preterm deliveries, as a result of chronic hypertension were prevented. Conclusions: Chronic hypertensive pregnancies are associated with increased risk for perinatal mortality. Planned early term delivery and targeting modifiable risk factors for chronic hypertension may reduce perinatal mortality rates.
\end{abstract}

Chronic Hypertension, Perinatal Mortality, and the Impact of Preterm Delivery:

A Population-Based Study in the United States, 2015-2018

Sonal Grover, MD, ${ }^{1}$ Justin S. Brandt, MD, ${ }^{2}$ Uma M. Reddy, MD, MPH ${ }^{3}$ Cande V. Ananth, PhD, MPH ${ }^{4-7}$

\section{Affiliations}

1. Department of Obstetrics, Gynecology and Reproductive Sciences, Rutgers Robert Wood Johnson Medical School, New Brunswick, NJ

2. Division of Maternal-Fetal Medicine, Department of Obstetrics, Gynecology and Reproductive Sciences, Rutgers Robert Wood Johnson Medical School, New Brunswick, NJ

3. Division of Maternal-Fetal Medicine, Department of Obstetrics, Gynecology, and Reproductive Sciences, Yale University School of Medicine, New Haven, CT

4. Division of Epidemiology and Biostatistics, Department of Obstetrics, Gynecology and Reproductive Sciences, Rutgers Robert Wood Johnson Medical School, New Brunswick, NJ 
5. Cardiovascular Institute of New Jersey, Rutgers Robert Wood Johnson Medical School, New Brunswick, NJ

6. Environmental and Occupational Health Sciences Institute (EOHSI), Rutgers Robert Wood Johnson Medical School, New Brunswick, NJ

7. Department of Biostatistics and Epidemiology, Rutgers School of Public Health, Piscataway, NJ

\section{Correspondence}

Cande V. Ananth, PhD, MPH

Division of Epidemiology and Biostatistics

Department of Obstetrics, Gynecology, and Reproductive Sciences

Rutgers Robert Wood Johnson Medical School

125 Paterson Street, New Brunswick NJ 08901

Email:ananthcv@rwjms.rutgers.edu

\section{ORCID ID}

Sonal Grover 0000-0003-4272-4114

Justin S. Brandt 0000-0002-3194-1087

Uma M. Reddy 0000-0002-8599-6040

Cande V. Ananth 0000-0002-0410-2595

Short Title: Chronic hypertension and perinatal mortality

Word counts : Abstract: 250; Manuscript: 2728

Figures: 1; Tables: 3

\section{Abstract}

Objective : To estimate the association between chronic hypertension and perinatal mortality and evaluate the extent to which this risk is impacted by preterm delivery.

Design: Cross-sectional analysis.

Setting: US, 2015-2018.

Population: Singleton births from 20-44 weeks' gestation.

Main outcomes and measures : We derived the risk of perinatal mortality in relation to chronic hypertension from fitting log-linear Poisson models with robust variance. Risk ratios (RR) and $95 \%$ confidence intervals (CI) were estimated after adjusting for confounders. The impact of misclassifications and unmeasured confounding biases were assessed. Causal mediation analysis was performed to quantify the impact of preterm delivery on the association.

Results: Of the $15,090,678$ singleton births, perinatal mortality was 22.5 per 1000 births in chronic hypertensive pregnancies compared to 8.2 per 1000 births in normotensive pregnancies (adjusted RR 2.05, 95\% CI 2.00, 2.10). Corrections for exposure misclassification and unmeasured confounding biases substantially increased the risk estimate. Although, causal mediation analysis revealed that most of the effect of chronic hypertension on perinatal mortality was mediated through preterm delivery, the perinatal mortality rates were highest at early term, term, and late term gestations, suggesting that a planned early term delivery at $37-38^{6 / 7}$ weeks may optimally balance risk in these pregnancies. Additionally, $87 \%(95 \%$ CI 84,90$)$ of perinatal deaths could be eliminated if preterm deliveries, as a result of chronic hypertension were prevented. 
Conclusions: Chronic hypertensive pregnancies are associated with increased risk for perinatal mortality. Planned early term delivery and targeting modifiable risk factors for chronic hypertension may reduce perinatal mortality rates.

\section{Tweetable Abstract:}

Maternal chronic hypertension is associated with increased risk for perinatal mortality, largely driven by preterm birth.

\section{Sources of Funding}

None.

Keywords : Chronic hypertension, perinatal mortality, stillbirth, neonatal death, preterm delivery; causal mediation analysis

\section{Introduction}

Chronic hypertension complicates 1-5\% of pregnancies, but there has been 13-fold increase in the prevalence of chronic hypertension in the United States (US) in the last four decades (1). The American obesity epidemic $(2,3)$, and deferred childbearing leading to older age of pregnant people, $(4,5)$ contribute substantially to this trend (6). Chronic hypertension during pregnancy is associated with increased rates of maternal complications, including death (7), superimposed preeclampsia (8, 9), placental abruption (10-12), end-stage renal disease (13), and long-term cardiovascular and cerebrovascular disease (12, 14, 15). Chronic hypertension is also an independent risk factor for an array of perinatal complications, including stillbirth,(16-18) fetal growth restriction,(19) neonatal death,(20,21) as well as neurological and neurodevelopmental deficits in children.(22-24) In light of the increasing prevalence rates of chronic hypertension and the associated complications, there is a need to evaluate the impact of chronic hypertension on perinatal mortality in the US, including stillbirth and neonatal death.

Chronic hypertension is associated with increased risk of preterm delivery,(8) and gestational age at delivery impacts neonatal outcomes in pregnancies complicated by chronic hypertension.(25) Gestational age also influences obstetrical care including interventions to improve neonatal outcomes (i.e., the administration of antenatal corticosteroids to promote fetal lung maturity) as well as delivery timing.(26) In the pathway between chronic hypertension and perinatal outcomes, preterm delivery features as an important component in the causal pathway, but how and, to what extent, preterm delivery plays a role in shaping these associations remain unknown.

We undertook a population-based cross-sectional study in a large contemporary US cohort to quantify the association between chronic hypertension and perinatal mortality and to disentangle the impact of preterm delivery as mediator. We hypothesized that chronic hypertension is associated with perinatal mortality and that preterm delivery plays an important role that mediates this causal association.

\section{Methods}

Study design and data sources

We performed a cross-sectional analysis of all singleton births in the US from 2015-2018. We utilized data on fetal deaths, live births, and linked live births-infant death records ascertained by the National Center for Health Statistics (NCHS) of Centers for Disease Control and Prevention. The live birth-infant death data is derived from infant death certificates linked to the live birth certificates for infants that died before reaching 1 year of age. Study variables were derived from the 2003 revision of the US standard certificates of vital events. Gestational age in these data were based on the best obstetrical estimate,(27) which had a $99.6 \%$ agreement within 2 weeks in comparison to data abstracted from hospital charts.(28) Since this de-identified data is publicly available, no ethics approval from an Institutional Review Board was sought. The study followed the STROBE reporting guidelines for cross-sectional studies.

Cohort composition 
The study population included all women who delivered singleton births in the US between 20-44 weeks' gestation from 2015-2018. We excluded pregnancies with multiple gestations, and records with missing gestational age or gestational age $<20$ weeks and [?] 45 weeks, as well as pregnancies with missing chronic hypertension status. After all the exclusions, 15,090,678 singleton births were included in the study (Supplemental Figure 1 ).

Exposure

The exposure was chronic hypertension as indicated on the US standard certificate of fetal death, live birth, and infant death. The diagnosis includes elevated blood pressure prior to pregnancy or recognized within the first 20 weeks of gestation.

\section{Outcomes measures}

The primary outcome was perinatal mortality, defined as a composite of stillbirth (fetal deaths at 20 weeks' gestation or greater), early neonatal death (deaths within 0-6 days of life), and late neonatal death (deaths between 7-28 days of life). Since timely obstetrical interventions may prevent stillbirth, but could lead to neonatal death, the composite was intended to capture perinatal mortalities that could be attributed to exposure to chronic hypertension. We also quantified the impact of chronic hypertension on stillbirth, as well as early and late neonatal deaths, as secondary outcomes.

\section{Statistical analyses}

We estimated perinatal mortality rates, expressed per 1000 births, for chronic hypertensive versus normotensive pregnancies. We examined the association between chronic hypertension and perinatal mortality on both the additive (risk difference, RD) and multiplicative scales (risk ratio, RR), derived from fitting log-linear Poisson regression models with robust variance and the identity-link for RD and log-link for RR (29). Associations with their corresponding $95 \%$ confidence intervals (CI) were derived before and after adjusting for confounders. We also examined associations between chronic hypertension and the risks of stillbirth, early neonatal deaths, and late neonatal deaths.

\section{Confounders}

We adjusted for several confounders including maternal age $(<15,15-19,20-24,25-29$ [reference], 30-34, 3539 , and [?] 40 years), education (below high school, up to high school, college, and beyond college educated), race/ethnicity (non-Hispanic White, non-Hispanic Black, Hispanic, or other race), maternal smoking (nonsmoker [reference], smoked before pregnancy only, and smoked before and during pregnancy), single marital status, pre-pregnancy body-mass index $\left(\mathrm{BMI}\right.$ in $\mathrm{kg} / \mathrm{m}^{2}$; underweight $[<18.5]$, normal [18.5-24.9], overweight [25.0-29.9], obese [30.0-34.9], and morbidly obese [[?]35.0]), and pregestational diabetes. We also adjusted for year of delivery to address potential temporal changes in the prevalence of chronic hypertension and maternal mortality.

\section{Missing data}

Since some of the covariates contained missing data, we imputed missing data through multiple imputation by chained equations approach.(30) We assumed that the pattern of missing data was "missing at random," and created 20 imputed data sets (after 25 burn-in iterations). All analyses were performed for each of the 20 data sets, and we combined the results of the analyses of imputations based on Rubin's principles.(31) Details regarding missing covariate data are shown in Supplemental Table 1 .

\section{Mediation analysis}

Gestational age features on the causal pathway between chronic hypertension and perinatal mortality as an mediator (Supplemental Figure 2 ); therefore, any adjustment for preterm delivery in the assessment of the perinatal mortality risk in relation to chronic hypertension would lead to collider bias.(32) To resolve this issue, we undertook a causal mediation analysis based on the counterfactual framework(33,34) to decompose the total effect (TE) of chronic hypertension on perinatal mortality into the natural direct effect (NDE; the 
effect of the exposure [chronic hypertension] on the outcome [perinatal mortality] if the mediator [preterm delivery $<37$ weeks] were set to what it would have been, probably contrary to the fact, in the absence of the exposure) and the natural indirect effect (NIE; the effect of the exposure on the outcome when the exposure is present after setting the mediator to what it would have been, probably contrary to the fact, with versus without the exposure).(35, 36) We also estimated the controlled direct effect (CDE; the effect of chronic hypertension on mortality that is not mediated through preterm delivery). The TE, NDE, NIE and CDE were estimated on multiplicative scale with RR and 95\% CI as effect measure.

In addition, we estimated the proportion mediated (PM; the proportion of the TE that is mediated through preterm delivery), and the proportion eliminated (PE; the proportion of the chronic hypertension-perinatal mortality association that might be eliminated by blocking the effect of chronic hypertension on preterm delivery). The $\mathrm{PE}$ is the proportion of perinatal deaths that could be prevented by designing interventions to reduce preterm delivery associated with chronic hypertension. All 95\% CI estimates were based on 1000 bootstrap samples.

\section{Sensitivity analysis: Probabilistic bias analysis}

Chronic hypertension is subject to misclassification in these data files, with sensitivity of $44 \%$ (and agreement of $98 \%$ ) in comparison to data abstracted from hospital medical charts.(28) To address potential exposure misclassification and unmeasured confounding biases, we undertook a sensitivity analysis through a probabilistic bias analysis. $(37,38)$ Exposure (chronic hypertension) misclassification was assumed to be differential with respect to the outcome (perinatal mortality). Based on a uniform distribution, we assumed the priors for sensitivity for chronic hypertension to range between 0.30 and 0.95 among those with perinatal deaths and 0.20 to 0.90 among live births; the priors for specificity for chronic hypertension was assumed to range between 0.98 and 1.00 both for deaths and live births, respectively.

Corrections for unmeasured confounding bias was based on the following assumptions: (i) we assumed that the prevalence of the unmeasured confounder among those with and without chronic hypertension, under a log-normal distribution, ranged between $5 \%$ and $15 \%$, and $3 \%$ and $10 \%$, respectively; and (ii) the RR for the confounder-outcome association was varied between 1.25 and 3.00. Under these assumptions, we drew the bias parameters 100,000 times from the prior distributions to address exposure misclassification and unmeasured confounding (computational strategy are provided in the R package "episenr".(39)) From these analyses, we report the median bias-corrected $\mathrm{RR}\left(\mathrm{RR}_{\mathrm{bc}}\right)$ and $95 \% \mathrm{CI}_{\mathrm{bc}}$.

Log-linear regression models and the mediation analysis were fit in SAS (version 9.4; SAS Institute, Cary, NC) using the GENMOD and the CAUSALMED procedures, respectively. The probabilistic bias analysis was implemented in R (R Foundation for Statistical Computing, Vienna, Austria) using the episensr package.(39)

\section{Results}

During the study period (2015-2018), there were 15,090,678 singleton births delivered between 20-44 weeks' gestation in the US (Supplemental Figure 1). The prevalence rate of chronic hypertension was 1.8\% $(\mathrm{n}=274,125)$. The distribution of socioeconomic factors in relation to chronic hypertension are shown inSupplemental Table 1 . Women with chronic hypertension were more likely to be older, African American, of higher parity, college educated, obese, and tobacco smokers, compared to normotensive women.

Perinatal mortality rates were higher among chronic hypertensive women (22.5 per 1000 births) compared to normotensive women (8.2 per 1000 births; Table 1 ). Among chronic hypertensive pregnancies, the rate of mortality was highest for stillbirth (16.1 per 1000 births), in comparison to early (4.6 per 1000 births) and late neonatal deaths (1.8 per 1000 births). Gestational age-specific risk of perinatal mortality (per 1000 births) among normotensive and chronic hypertensive pregnancies are described in Figure 1 . These rates were particularly high at early term, term, and late term gestations

The absolute risks and $\mathrm{RR}$ of perinatal mortality in pregnancies complicated by chronic hypertension are described in Table 2 . After adjusting for potential confounders, chronic hypertension was associated with 11.0 (95\% CI 10.5, 11.5) per 1000 excess perinatal deaths over the normotensive group. The increase in risk 
of perinatal death in chronic hypertensive pregnancies was two-fold higher (adjusted RR 2.05, 95\% CI 2.00, 2.10), compared to normotensive pregnancies.

\section{Sensitivity analysis}

The bias-corrected RRs, with simultaneous corrections for both exposure misclassification and unmeasured confounding, revealed the risk of perinatal mortality in chronic hypertensive pregnancies was almost five-fold higher $\left(\mathrm{RR}_{\mathrm{bc}} 4.86,95 \%\right.$ CI 3.42, 5.98), compared to normotensive pregnancies. The risk was highest for stillbirth in relation to chronic hypertension $\left(\mathrm{RR}_{\mathrm{bc}} 7.76,95 \%\right.$ CI $\left.4.52,11.12\right)$.

\section{Causal mediation analysis}

The causal mediation analysis was performed to disentangle the total effect of chronic hypertension-perinatal mortality association, with preterm delivery as the potential mediator (Table 3 ). Much of the TE of perinatal mortality and its component mortalities was mediated through preterm delivery. The indirect effect was greatest for perinatal mortality and stillbirth. Given the strong mediation component of the chronic hypertension-mortality association that operates through preterm delivery, the PE estimate is correspondingly high: $87 \%$ (95\% CI 84, 90).

\section{Discussion}

\section{Principal findings}

In this population-based study that included over 15 million singleton births in the US from 2015-2018, we found that chronic hypertension was associated with substantially increased rates of perinatal mortality, including stillbirth and neonatal deaths. The causal mediation analysis suggested that the major driver of mortality risk in chronic hypertensive pregnancies was mediated through preterm delivery. There was also higher risk of perinatal mortality after 39 weeks' gestation. These results suggest that a planned, early term delivery at $37-38^{6 / 7}$ weeks may optimally balance risk in these pregnancies.

\section{Interpretation}

An understanding of the perinatal risks associated with chronic hypertension is largely informed by older studies. For example, a prior population based study from the US, which utilized Nationwide Inpatient Sample data from 1995-2008, reported a higher risk for stillbirth (odds ratio [OR] 2.31, 95\% CI 2.11, 2.53) for pregnancies complicated by chronic hypertension compared to normotensive pregnancies.(6) In a populationbased prospective cohort study from Sweden (1992-2004), an increase in stillbirth risk (OR 2.71, 95\% CI 1.96, 3.73) and neonatal death (OR 2.89, 95\% CI 1.95, 4.30) was observed.(21) The results of our study, which showed increased perinatal mortality associated with chronic hypertension, are consistent with these studies, yet the magnitude of risk was larger. After adjusting for bias due to misclassification and unmeasured confounders, we found the risk of perinatal mortality was 4.86 (95\% CI 3.42, 5.98), which was largely due to stillbirth at term gestations. The reason for this difference is uncertain, but may be due to advances in neonatal care that minimize the risk of neonatal deaths.

The mediation analysis suggested that a substantial driver of perinatal mortality risk is preterm delivery. Although most deliveries in our cohort occurred at term, there was an association between chronic hypertension and preterm delivery. A prior population based study from the US found the odds of preterm delivery for chronic hypertensive versus normotensive pregnancies was 3.01 (95\% CI 2.88, 3.14).(6) A meta-analysis on chronic hypertension and pregnancy outcomes showed that pregnancies complicated by chronic hypertension are at a higher risk of preterm delivery <37 weeks' gestation (RR 2.7, 95\% CI 1.9, 36).(8) Most often the preterm births are medically indicated secondary to superimposed preeclampsia or fetal growth restriction. $(40,41)$ This study suggests that, if possible, avoiding preterm delivery in pregnancies complicated by chronic hypertension may have large impact on perinatal mortality.

The results of our study are consistent with prior work(42) and professional society recommendations(43) that suggest the optimal timing of delivery for pregnancies complicated by chronic hypertension is 37-38 weeks. It is critical to avoid early preterm delivery in these pregnancies (due to preterm delivery-associated 
complications and long-term chronic health conditions), but term and late term pregnancies also carry high risk of stillbirth. Despite national efforts to avoid early term deliveries,(44) findings from this study suggest early term deliveries may minimize the risks of stillbirths associated with chronic hypertension.

Women with chronic hypertension often have risk factors, such as advanced maternal age, obesity, and tobacco smoking, $(1,45,46)$ which, in turn, increase the risks of adverse perinatal outcomes.(47-51) Public health strategies that target modifiable risk factors to reduce the burden of chronic hypertension may be the best strategy to reduce perinatal mortality associated with this disease. The mediation analysis quantified the preventable proportion of perinatal deaths associated with chronic hypertension by eliminating preterm deliveries associated with chronic hypertension, and most of perinatal deaths could be avoided.

While this study improves our understanding of the magnitude of chronic hypertension's impact on perinatal mortality, there is a need for further study: (i) to develop preventive strategies such as weight loss and preconception blood pressure management for women at risk; (ii) to assess the effectiveness of these strategies on perinatal outcomes; and (iii) to evaluate whether treating mild chronic hypertension in pregnancy has the potential to reduce the risk of adverse pregnancy outcomes.(52)

\section{Strengths and weaknesses}

The main strengths of the study were that it was large and contemporary. This not only increases the generalizability of the findings, but also reflects current practices in the US. In addition, while we quantified the association between chronic hypertension and perinatal mortality, we also performed a causal mediation analysis to evaluate the impact of preterm delivery on this association. An application of causal inference methodology in this context provides insight into what drives the risk, which can be used to generate novel approaches to reduce the risks associated with chronic hypertension.

Despite these strengths, the study as some limitations. There may be a difference in risk for pregnancies complicated by mild chronic hypertension versus pregnancies complicated by severe disease that require antihypertensive medications, but we lacked data on the severity of chronic hypertension. There is also possible misclassification bias due to inaccuracy in recording diagnosis of chronic hypertension in the data and inability to distinguish women who may have developed superimposed preeclampsia. We have attempted to account for these misclassification and unmeasured confounder biases in the sensitivity analysis.

\section{Conclusions}

Compared to normotensive pregnancies, pregnancies complicated by chronic hypertension are associated with increased risk for perinatal mortality, including stillbirth and neonatal death. A large driver of this risk was preterm delivery, but there was also a substantial increased risk of perinatal mortality at term and late term gestations. The results of this study suggest the best strategies to reduce the risk of perinatal mortality include planned, early term deliveries and targeting modifiable risk factors for chronic hypertension, such as advanced maternal age and obesity, that may impact the prevalence of chronic hypertension.

\section{Acknowledgements}

None.

\section{Disclosures of Interests}

All authors declare no support from any organization for the submitted work; no financial relationships with any organizations that might have an interest in the submitted work in the previous three years; and no other relationships or activities that could appear to have influenced the submitted work.

\section{Contribution of Authorship}

Sonal Grover: Drafting of the manuscript.

Justin S. Brandt: Reviewing and editing the manuscript.

Uma M. Reddy: Reviewing and editing the manuscript. 
Cande V. Ananth: Reviewing and editing the manuscript, and performing all statistical analysis

\section{Details of Ethics Approval}

None.

\section{Funding}

None.

\section{References}

1. Ananth CV, Duzyj CM, Yadava S, Schwebel M, Tita ATN, Joseph KS. Changes in the Prevalence of Chronic Hypertension in Pregnancy, United States, 1970 to 2010. Hypertension. 2019;74(5):1089-95.

2. Kim SY, Dietz PM, England L, Morrow B, Callaghan WM. Trends in pre-pregnancy obesity in nine states, 1993-2003. Obesity (Silver Spring). 2007;15(4):986-93.

3. Hales CM, Fryar CD, Carroll MD, Freedman DS, Ogden CL. Trends in Obesity and Severe Obesity Prevalence in US Youth and Adults by Sex and Age, 2007-2008 to 2015-2016. JAMA. 2018;319(16):1723-5.

4. Martin JA, Hamilton BE, Ventura SJ, Osterman MJ, Kirmeyer S, Mathews TJ, et al. Births: final data for 2009. Natl Vital Stat Rep. 2011;60(1):1-70.

5. Misra DP, Ananth CV. Infant mortality among singletons and twins in the United States during 2 decades: effects of maternal age. Pediatrics. 2002;110(6):1163-8.

6. Bateman BT, Bansil P, Hernandez-Diaz S, Mhyre JM, Callaghan WM, Kuklina EV. Prevalence, trends, and outcomes of chronic hypertension: a nationwide sample of delivery admissions. Am J Obstet Gynecol. 2012;206(2):134 e1-8.

7. Creanga AA, Berg CJ, Syverson C, Seed K, Bruce FC, Callaghan WM. Pregnancy-related mortality in the United States, 2006-2010. Obstet Gynecol. 2015;125(1):5-12.

8. Bramham K, Parnell B, Nelson-Piercy C, Seed PT, Poston L, Chappell LC. Chronic hypertension and pregnancy outcomes: systematic review and meta-analysis. BMJ. 2014;348:g2301.

9. Sutton EF, Hauspurg A, Caritis SN, Powers RW, Catov JM. Maternal Outcomes Associated With Lower Range Stage 1 Hypertension. Obstet Gynecol. 2018;132(4):843-9.

10. Williams MA, Mittendorf R, Monson RR. Chronic hypertension, cigarette smoking, and abruptio placentae. Epidemiology. 1991;2(6):450-3.

11. Ananth CV, Vintzileos AM. Ischemic placental disease: epidemiology and risk factors. Eur J Obstet Gynecol Reprod Biol. 2011;159(1):77-82.

12. Wu P, Chew-Graham CA, Maas AH, Chappell LC, Potts JE, Gulati M, et al. Temporal Changes in Hypertensive Disorders of Pregnancy and Impact on Cardiovascular and Obstetric Outcomes. Am J Cardiol. 2020;125(10):1508-16.

13. Wu CC, Chen SH, Ho CH, Liang FW, Chu CC, Wang HY, et al. End-stage renal disease after hypertensive disorders in pregnancy. Am J Obstet Gynecol. 2014;210(2):147 e1-8.

14. Cirillo PM, Cohn BA. Pregnancy complications and cardiovascular disease death: 50-year follow-up of the Child Health and Development Studies pregnancy cohort. Circulation. 2015;132(13):1234-42.

15. Grandi SM, Filion KB, Yoon S, Ayele HT, Doyle CM, Hutcheon JA, et al. Cardiovascular DiseaseRelated Morbidity and Mortality in Women With a History of Pregnancy Complications. Circulation. 2019;139(8):1069-79. 
16. Ankumah NA, Cantu J, Jauk V, Biggio J, Hauth J, Andrews W, et al. Risk of adverse pregnancy outcomes in women with mild chronic hypertension before 20 weeks of gestation. Obstet Gynecol. 2014;123(5):966-72.

17. Ananth CV, Savitz DA, Bowes WA, Jr. Hypertensive disorders of pregnancy and stillbirth in North Carolina, 1988 to 1991. Acta Obstet Gynecol Scand. 1995;74(10):788-93.

18. Panaitescu AM, Syngelaki A, Prodan N, Akolekar R, Nicolaides KH. Chronic hypertension and adverse pregnancy outcome: a cohort study. Ultrasound Obstet Gynecol. 2017;50(2):228-35.

19. Nzelu D, Dumitrascu-Biris D, Kay P, Nicolaides KH, Kametas NA. Severe hypertension, preeclampsia and small for gestational age in women with chronic hypertension diagnosed before and during pregnancy. Pregnancy Hypertens. 2018;14:200-4.

20. Ananth CV, Basso O. Impact of pregnancy-induced hypertension on stillbirth and neonatal mortality. Epidemiology. 2010;21(1):118-23.

21. Zetterstrom K, Lindeberg SN, Haglund B, Hanson U. The association of maternal chronic hypertension with perinatal death in male and female offspring: a record linkage study of 866,188 women. BJOG. 2008;115(11):1436-42.

22. Tuovinen S, Eriksson JG, Kajantie E, Lahti J, Pesonen AK, Heinonen K, et al. Maternal hypertensive disorders in pregnancy and self-reported cognitive impairment of the offspring 70 years later: the Helsinki Birth Cohort Study. Am J Obstet Gynecol. 2013;208(3):200 e1-9.

23. Tuovinen S, Aalto-Viljakainen T, Eriksson JG, Kajantie E, Lahti J, Pesonen AK, et al. Maternal hypertensive disorders during pregnancy: adaptive functioning and psychiatric and psychological problems of the older offspring. BJOG. 2014;121(12):1482-91.

24. Grace T, Bulsara M, Pennell C, Hands B. Maternal hypertensive diseases negatively affect offspring motor development. Pregnancy Hypertens. 2014;4(3):209-14.

25. Harper LM, Biggio JR, Anderson S, Tita AT. Gestational Age of Delivery in Pregnancies Complicated by Chronic Hypertension. Obstet Gynecol. 2016;127(6):1101-9.

26. American College of Obstetricians Gynecologists. Hypertension in pregnancy. Report of the American College of Obstetricians and Gynecologists' Task Force on Hypertension in Pregnancy. Obstet Gynecol. 2013;122(5):1122-31.

27. Martin JA, Osterman MJ, Kirmeyer SE, Gregory EC. Measuring Gestational Age in Vital Statistics Data: Transitioning to the Obstetric Estimate. Natl Vital Stat Rep. 2015;64(5):1-20.

28. Gregory ECW, Martin JA, Argov EL, Osterman MJK. Assessing the Quality of Medical and Health Data From the 2003 Birth Certificate Revision: Results From New York City. Natl Vital Stat Rep. 2019;68(8):120.

29. Spiegelman D, Hertzmark E. Easy SAS calculations for risk or prevalence ratios and differences. Am J Epidemiol. 2005;162(3):199-200.

30. Harel O, Mitchell EM, Perkins NJ, Cole SR, Tchetgen Tchetgen EJ, Sun B, et al. Multiple Imputation for Incomplete Data in Epidemiologic Studies. Am J Epidemiol. 2018;187(3):576-84.

31. Rubin DB. Inference and missing data. Biometrika. 1976;63(3):581-92.

32. Ananth CV, Schisterman EF. Confounding, causality, and confusion: the role of intermediate variables in interpreting observational studies in obstetrics. Am J Obstet Gynecol. 2017;217(2):167-75.

33. Robins JM, Greenland S. Identifiability and exchangeability for direct and indirect effects. Epidemiology. 1992;3(2):143-55. 
34. Pearl J. Direct and Indirect Effects. Proceedings of the Seventeenth conference on Uncertainty in artificial intelligence. 2001:411-20.

35. Valeri L, Vanderweele TJ. Mediation analysis allowing for exposure-mediator interactions and causal interpretation: theoretical assumptions and implementation with SAS and SPSS macros. Psychol Methods. 2013;18(2):137-50.

36. Ananth CV, VanderWeele TJ. Placental abruption and perinatal mortality with preterm delivery as a mediator: disentangling direct and indirect effects. Am J Epidemiol. 2011;174(1):99-108.

37. Lash TL, Fox MP, Fink AK. Applying quantitative bias analysis to epidemiologic data.: Springer-Verlag New York; 2009.

38. Lash TL, Fox MP, MacLehose RF, Maldonado G, McCandless LC, Greenland S. Good practices for quantitative bias analysis. Int J Epidemiol. 2014;43(6):1969-85.

39. Episensr HD. Basic Sensitivity Analysis of Epidemiological Results. R package 0.9.999. ed2020.

40. Premkumar A, Baer RJ, Jelliffe-Pawlowski LL, Norton ME. Hypertensive Disorders of Pregnancy and Preterm Birth Rates among Black Women. Am J Perinatol. 2019;36(2):148-54.

41. Kase BA, Carreno CA, Blackwell SC, Sibai BM. The impact of medically indicated and spontaneous preterm birth among hypertensive women. Am J Perinatol. 2013;30(10):843-8.

42. Hutcheon JA, Lisonkova S, Magee LA, Von Dadelszen P, Woo HL, Liu S, et al. Optimal timing of delivery in pregnancies with pre-existing hypertension. BJOG. 2011;118(1):49-54.

43. American College of Obstetricians Gynecologists, Committee on Practice Bulletins, Obstetrics. ACOG Practice Bulletin No. 203: Chronic Hypertension in Pregnancy. Obstet Gynecol. 2019;133(1):e26-e50.

44. Oshiro BT, Kowalewski L, Sappenfield W, Alter CC, Bettegowda VR, Russell R, et al. A multistate quality improvement program to decrease elective deliveries before 39 weeks of gestation. Obstet Gynecol. 2013;121(5):1025-31.

45. Niskanen L, Laaksonen DE, Nyyssonen K, Punnonen K, Valkonen VP, Fuentes R, et al. Inflammation, abdominal obesity, and smoking as predictors of hypertension. Hypertension. 2004;44(6):859-65.

46. Gurven M, Blackwell AD, Rodriguez DE, Stieglitz J, Kaplan H. Does blood pressure inevitably rise with age?: longitudinal evidence among forager-horticulturalists. Hypertension. 2012;60(1):25-33.

47. Chen A, Feresu SA, Fernandez C, Rogan WJ. Maternal obesity and the risk of infant death in the United States. Epidemiology. 2009;20(1):74-81.

48. Jacobsson B, Ladfors L, Milsom I. Advanced maternal age and adverse perinatal outcome. Obstet Gynecol. 2004;104(4):727-33.

49. Delbaere I, Verstraelen H, Goetgeluk S, Martens G, De Backer G, Temmerman M. Pregnancy outcome in primiparae of advanced maternal age. Eur J Obstet Gynecol Reprod Biol. 2007;135(1):41-6.

50. McDonald SD, Han Z, Mulla S, Beyene J, Knowledge Synthesis G. Overweight and obesity in mothers and risk of preterm birth and low birth weight infants: systematic review and meta-analyses. BMJ. 2010;341:c3428.

51. Pineles BL, Hsu S, Park E, Samet JM. Systematic Review and Meta-Analyses of Perinatal Death and Maternal Exposure to Tobacco Smoke During Pregnancy. Am J Epidemiol. 2016;184(2):87-97.

52. NIH. Chronic Hypertension and Pregnancy (CHAP) Project. 2020.

\section{Hosted file}


chr-pnd-tables figures.pdf available at https://authorea.com/users/400682/articles/ 512971-chronic-hypertension-perinatal-mortality-and-the-impact-of-preterm-delivery-apopulation-based-study-in-the-united-states-2015-2018 